\title{
Effective Procedure to Predict Rainfall Conditions using Hybrid Machine Learning Strategies
}

\author{
S.Sakthivel ${ }^{1}$, Dr.(Mrs.) G.Thailambal² \\ ${ }^{1} \mathrm{Ph} . \mathrm{D}$ Research Scholar, Vels Institute of Science, Technology and Advanced Studies (VISTAS),Chennai, \\ India, sakthivelphd78@gmail.com. \\ ${ }^{2}$ Research Supervisor, Vels Institute of Science, Technology and Advanced Studies (VISTAS),Chennai, India, \\ thaila.scs@velsuniv.ac.in.
}

Article History:Received:11 november 2020; Accepted: 27 December 2020; Published online: 05 April 2021

\begin{abstract}
In the present information technology stream supports many natural disaster prediction schemes to save several people from disaster scenarios. In such case, rainfall prediction and analysis is the most important concern to take care as well as the prediction of high rainfall saves many individual's life and their assets. This kind of rainfall prediction schemes provides a facilitation to take respective precautions to avoid huge damages further. The rainfall predictions are categorized into two different variants such as Limited Period Rainfall Prediction and the long period Continuous Rainfall Prediction. Several past analysis and literatures provide accurate predictions for limited period rainfall but the major problem is to identify or predict the continuous long period rainfall. This kind of drawbacks leads many researchers to work on this domain and predict the rainfall status exactly for both limited period as well as long period continues rainfall. In this paper, a new hybrid machine learning strategy is implemented to predict the rainfall status exactly, in which the proposed methodology is named as Intense Neural Network Mining (INNM). This proposed approach of INNM analyze the rainfall prediction scenario based on two different machine learning logics such as Back Propagation Neural Network and the Rapid Miner. The general machine learning algorithms train the machine with respect to the dataset features and predict the result based on testing input. In this approach two different variants of machine learning principles are utilized to classify the resulting nature with better accuracy levels and crossvalidations are providing best probabilistic results in outcome. And these two logics are integrated together to produce a new hybrid machine learning strategy to predict the rainfall status exactly and save human life against disasters. In this paper, a novel dataset is utilized from Regional Meteorological Centre Chennai to predict the rainfall summary in clear manner and the summarization of specific dataset is described on further sections. The proposed approach of INNM assures the resulting accuracy levels around $96.5 \%$ in prediction with lowest error ratio of $0.04 \%$ and the resulting portion of this paper provides a proper proof of this outcome in graphical manner.
\end{abstract}

Keywords: Rainfall Identification, Hybrid Machine Learning, Intense Neural Network Mining, INNM, Back Propagation Neural Network, Rapid Miner

\section{INTRODUCTION}

The Prediction Strategies are the most important concern to all field in both commercial and non-commercial sectors to develop their business, improve the selling opportunities, natural disasters and more. In specific natural disaster predictions are the most significant prediction scheme to deal with several human lives, so that many researchers work on the field of disaster prediction logics. A different gathering of researchers from everywhere in the world has created stochastic climate models with the assistance of Random Number Generator, which can deliver a yield that looks like the climate information, helping them to construct a gigantic dataset with no need of manual chronicle. In these disaster prediction schemes rainfall prediction is the most important need now-a-days, because in the year of 2021 India met a heavy rainfall in several states and attain a huge destruction level in most of the places [1][2]. These causes raise an intention to develop a new intelligent approach to predict the rainfall levels exactly and do some precautions accordingly. India is a country that comprises of in excess of a billion people as well as more than $80 \%$ of this populace are needy upon agriculture based works professionally. The uncommon field of rainfall level prediction, which is essential for all individuals and the rainfall level prediction plays an imperative in forestalling causalities occurred because of natural-disaster scenarios [3].

This additionally causes us to keep up the water assets appropriately and exceptionally exact rainfall forecast is helpful if there should arises an occurrence of substantial rainfall which may cause flood and no rainfall

*Corresponding author: S.Sakthivel

209

Ph.D Research Scholar, Vels Institute of Science, Technology and Advanced Studies (VISTAS), Chennai, India, sakthivelphd78@gmail.com. 
which may make dry season keep up our water assets. In the nation like India, where yields are reaped dependent on the rainstorm season and along these lines, having progressed information in level prediction is significant. A few states are influenced by dry spells while some with floods as well as this forecast model can assist us with playing it safe to save lives by giving vehicle and food. To tackle these issues, different rainfall level prediction procedures are required to propose in proper manner [7][8]. In this paper, a novel hybrid machine learning strategy is implemented to attain high level of rainfall predictions in an intelligent manner with proper accuracy levels. The proposed approach is called Intense Neural Network Mining (INNM), in which it is acquired from two different machine learning techniques such as Back Propagation Neural Network and the Rapid Miner Logic. These two algorithms are integrated together and provide a proper support to predict the rainfall constraints in an efficient manner. The major intention of this proposed approach INNM is to create a new methodology to estimate the level of rainfall in prediction manner using machine learning principles without any hurdles in an outcome. Weather prediction logic is a complex task to deal with as well as it deserves some challenging skill set to acquire and manipulate the dataset with different proportions and attain the best outcome in resulting unit. The proposed approach utilizes the exact parameters and attributes, in which it is necessary to predict the rainfall during the particular monsoon. The major contributions of this paper are listed below in detail.

(i) To design a new machine learning strategy to provide an efficient prediction skill to the machine to identify the rainfall levels in an innovative way.

(ii) The main intention is to improve the accuracy levels on prediction drastically to support the farmers and people in good manner.

(iii) Process the dataset attribute values with high precision levels and create a training model with proper accuracy levels.

(iv) To provide better understanding regarding weather forecasting by means of simple graphical representation or numerical forecast equation.

In India the prediction levels are so restricted and the focus felt down with only high range of disasters such as Tsunami, flood and so on. The research level for identifying and predicting the monsoon levels need to be improved more in Tamil Nadu. The classical machine learning based prediction strategies looks for limited rainfall level predictions alone as well as the prediction necessities are more in this nation as compared to others, because in India more than $80 \%$ of people belonging to farming and agriculture. These two fields compulsorily require a rainfall as well as if the rainfall level is high these two fields are affected drastically, so that the prediction of rainfall level is must to preserver many people life [9][10]. The following are the societal contributions of this paper.

(i) Agricultural and Farming sectors and water supplies mostly rely upon the rainfall forecasting.

(ii) Rainfall varieties influence Indian economy on the grounds that the countries in excess of $80 \%$ of individuals dependent on agri-business and farming.

(iii) Rainfall level expectations provide a huge help to fishermen's to save their life on the ocean from cyclone conditions, heavy-storm scenarios and the gust.

(iv) Countries calamity group will find a way to save people groups from flood and substantial rainfall conditions.

These factors makes the rainfall prediction analysis scheme most important and the best way for predicting the rainfall conditions are machine learning strategies, in which the proposed approach utilizes the same from two different algorithms such as Back Propagation Neural Network and the Rapid Miner Logic to introduce a new machine learning model called INNM, in which it provides an accurate rainfall prediction modes with proper accuracy levels.

The rest of this paper describe regarding Related Study over section 2, further section of Section 3 illustrates the proposed system methodologies in detail with proper algorithm flow and the Section 4 illustrates the Result and Discussion portion of the paper and the final section, Section 5 illustrates the concept of Conclusion and Future Scope of the proposed paper. These all will be explained in detail over the further section summaries.

\section{RELATED STUDY}

In the year of 2020, the authors "XiaoboZhang et al. [4]" proposed a paper related to yearly rainfall level predictions as well as non-raining condition predictions with respect to Odisha state (India). In this paper [4], the authors described such as: rainfall is the natural activity, which is expected to get by every year on regular basis as well as this provides a major water resource to many countries, especially India. But the preservation of water is a main cause to save the rainfall resource in an innovative way. But without any proper predictions, it is hard to analyze the level of storage unit maintenance and complex to manage these things in rainfall stages. In this 
paper [4], a detailed study on Odisha Government rainfall prediction strategies with respect to yearly rainfall level estimations as well as non-raining climate estimations. The dataset of yearly rainfall status is accumulated for prediction of rainfall status in the Odisha state, in which the records of 1991 to 2015 are properly obtained to estimate the rainfall levels intensively by sing the machine learning strategy called Support Vector Regression with Multilayer Perception Network. This algorithm effectively worked and predicts the rainfall status with the help of following parameters such as: monthly temperature ratio, wind speed, humidity level, cloud status and non-raining days average. Based on these parameters the estimation of rainfall and the related predictions are done in an efficient manner with the help of machine learning algorithm called SVR-MLP [4].

In the year of 2020, the authors "RajibMaity et al. [5]" proposed a paper related to rainfall level predictions with respect to General-Circulation-Model and hybrid deep learning procedures. In this paper [5], the authors illustrated such as deep learning principles are strong enough to deal with some complex level applications. This paper [5] introduces a hybrid deep learning procedure to analyze the rainfall ratio based on annual estimations. This proposed hybrid algorithm [5] is an integrated approach, in which the 1-D Convolutional Neural Network (Conv1D) and the Multi-Layer Perception (MLP) are linked together to provide an efficient prediction strategy of the rainfall predictions. In this paper [5] 9 different estimation parameters are used to analyze the rainfall predictions, in which the metrics are attained from the General Circulation Model.

This paper [5] illustrates in many region such kind of rainfall prediction logics are still unavailable as well as the machine learning approaches are complex to initialize in such region rainfall estimations due to the long term rainfall ratio. To resolve the mentioned issues a deep learning principles are used over this paper to predict the rainfall levels accurately in daily as well as yearly manner. The concept of MLP is a deep learning procedure and the concept of SVR is the machine learning procedure, in which these two logics are integrated together to provide an effective rainfall identification port in this paper [5]. For all, the hybrid Deep learning principles are predicting the rainfall status effectively and this analysis [5] described that in a fine manner.

In the year of 2020, the authors "GuohuiLi et al. [6]" proposed a paper related to rainfall prediction analysis with respect to novel prediction replica. In this paper [6], the authors predict the rainfall summary of every month with proper error estimation principles. The prediction of rainfall is so important, in which it indirectly cases several damages in nature by means of its co-related effects such as flood, strom and so on as well as these cases illustrates the importance of rainfall level predictions with respect to monthly mean value analysis. This kind of optimization process eliminates the flaw over prediction of rainfall as well as in this paper [6] a new improved butterfly optimization logic is introduced to estimate the rainfall ratio more effectively. This new model is comprised with several classification and learning strategies such as: variation-Mode-Decomposition, Least Square SVM Classifier and ARMA replication. The graphical estimations over the resulting section of this paper [6] show that the proposed improved butterfly optimization logic is comparatively better than the classical approaches such as PSO, DE and so on. In this paper [6], the maximum error ratio attained while rainfall is not more than 9 millimeter and the overall accuracy levels are enhanced over $43 \%$ as compared to the previous models as well as this model creates a way to many researchers to think regarding the hybrid model.

\section{Proposed System Methodologies}

In this paper, a new hybrid prediction logic is designed called Intense Neural Network Mining (INNM) with the help of two different machine learning principles such as Back Propagation Neural Network (BPNN) and the Rapid Miner (RM). The proposed approach of INNM provides the highest accuracy range over prediction of rainfall with respect to machine learning based classification principles. The prediction accuracy ranges around $96.5 \%$ is attained with the help of this INNM approach with the lowest error ratio of $0.04 \%$ for analyzing the records ranges from 2000 to 2017 with the ranges of minimum 1500 to 200 records are obtained from every year rainfall data. This approach utilizes the data from the dataset provided by Chennai-Regional Meteorological Centre (C-RMC), in which the weather forecasting data and the related details are acquired from this dataset. These records are processed with the help of the proposed INNM logic and attain the mentioned accuracy levels with least error ratio. The nature of back propagation is to tune the neural network resource in fine manner with respect to the weight ratio of the obtained data in the past iterations (epochs). This provides high efficiency to the proposed Intense Neural Network Mining model with least error rates as well as the Rapid Miner logic is implied over the proposed approach of INNM to mine the rainfall data obtained from dataset based on the following three parameters such as information mining, text extraction and prediction estimation. The Rapid Miner is generally an open source free software library for estimating the data based on mining rules. All these prediction principles are clearly explained in detail over further summaries. The following figure, Fig-1 illustrates the clear view of proposed approach INNM architecture with all processing scenarios in detail. 


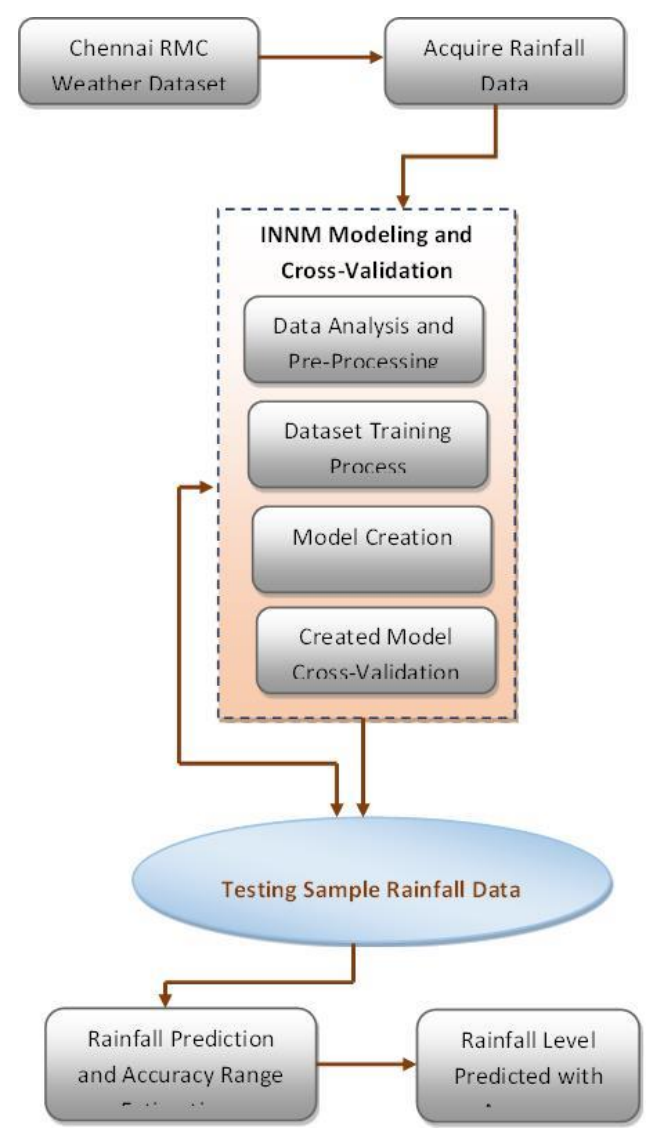

Fig.1 Proposed Approach INNM Architectural Design

\section{A. Data Analysis and Pre-Processing}

This data analysis process estimate the input data based on certain metric categorization with respect to number of rows and columns presented into the dataset. In this paper, five different parameters are considered for the prediction of rainfall status by using the proposed approach called INNM. The five parameter categorizations are: Rainfall Estimated Date, Location, Minimum Temperature, Maximum Temperature and the Rainfall Level. Based on these parameters the prediction process is happening and the performance measures are calculated with respect to back propagation laws. These different metrics are analyzed clearly to manipulate the performance ratio of rainfall in the respective location as well as train the model based on these estimated metrics.

The next level of data processing is handled by means of the principle followed by Rapid Miner logic, in which it handles the data pre-processing nature to make the rainfall dataset input as error-free. In this approach of the logic of reverse mapping is considered to reverse the processed system data into human visualization data, in which the reverse mapping principles are helpful to monitor the rainfall status in associated label structure. This pre-processing mode eliminates the error data in the dataset and provides the raw error-free data for processing in order to boolean results. The pre-processing logic and the data analysis estimations of rainfall data is manipulated based on the following equations (1) and (2).

$$
R_{D} F\{r, c\} \leftarrow \sum R^{M a p}\left(L\left[W^{\text {Score }}\right] \cdot \sum W D^{(I-n)}\right.
$$

Where the $\mathrm{R}_{\mathrm{D}} \mathrm{F}$ indicates the rainfall decision factor, ' $\mathrm{r}$ ' and ' $\mathrm{c}$ ' indicates the respective number of rows and columns in the input dataset, $\mathrm{R}^{\mathrm{Map}}$ indicates the Reverse Mapping logic, $\mathrm{L}$ indicates the reverse mapping label, $\mathrm{W}^{\text {Score }}$ indicates the weight score value, $W D$ indicates the weather data and the process is continued from 1 to $\mathrm{n}$ iteratively until the last value of the dataset is acquired.

$$
P^{R} \leftarrow R_{D} F\{r, c\} \cdot \sum_{i=0}^{n-1} W\left(\frac{P(x, y)-C(x, y)}{r M}\right)
$$


Where the $\mathrm{P}^{\mathrm{R}}$ indicates the Pre-processing outcome, $\mathrm{P}(\mathrm{x}, \mathrm{y})$ indicates the previous rainfall measures and $\mathrm{C}(\mathrm{x}, \mathrm{y})$ indicates the present rainfall measure taken from the dataset loop for processing, $\mathrm{W}$ indicates the weight factor and $\mathrm{rM}$ indicates the millimeter level of the rainfall. The following table, Table-1 shows the sample dataset values with respect to different location specifications [11].

Table-1: Sample Dataset Values

\begin{tabular}{|l|l|l|l|l|}
\hline Date & Location & $\begin{array}{l}\text { Minimum } \\
\text { Temperature }\end{array}$ & $\begin{array}{l}\text { Maximum } \\
\text { Temperature }\end{array}$ & $\begin{array}{l}\text { Rainfall } \\
(\mathbf{m m})\end{array}$ \\
\hline $1 / 1 / 2021$ & Ariyalur & 9.0 & 10.6 & 216.1 \\
\hline $1 / 2 / 2021$ & Erode & 10.2 & 15.9 & 58.7 \\
\hline $1 / 2 / 2021$ & Dindigul & 12.9 & 13.89 & 179.6 \\
\hline $1 / 2 / 2021$ & Karur & 15.32 & 16.76 & 109.1 \\
\hline $1 / 2 / 2021$ & Madurai & 10.332 & 10.658 & 131.2 \\
\hline $1 / 3 / 2021$ & Ranipet & 16.36 & 17.365 & 44.0 \\
\hline $1 / 3 / 2021$ & Salem & 16.96 & 18.02 & 53.4 \\
\hline $1 / 5 / 2021$ & Chennai & 7.65 & 10.328 & 194.4 \\
\hline $1 / 15 / 2021$ & Kariakal & 4.26 & 7.365 & 283.7 \\
\hline $1 / 17 / 2021$ & Theni & 10.385 & 12.698 & 109.9 \\
\hline $1 / 17 / 2021$ & Thenkasi & 8.365 & 9.134 & 124.1 \\
\hline
\end{tabular}

The following algorithm, Algorithm-1 demonstrates the complete process flow of the data analysis and the preprocessing stages in detail with proper Pseudocode.

\author{
Algorithm-1: Data Analysis and Preprocessing \\ Input: C-RMC Dataset \\ Output: Preprocessed Rainfall Data with Reverse Mapping Values
}

1. Call the required libraries for estimation such as num-py, and pandas_lib.

2. Acquire the dataset from the system drive or from net source, in which the dataset variables and values need to be merged by using 'merge' function.

3. Extract the number of rows and columns available into the dataset, in which the associated values are the data analysis metrics. These metrics are usually analyzed from the columns extracted from the dataset.

4. Analyze the weight score metric to the categorical format variable for manipulating the final boolean response (Ref. eqn(1)).

\title{
Pseudocode:
}

Import 'num-py' as np

Import 'pandas_lib' as pd

DS $\leftarrow \mathrm{rf}($ load_data $($ C-RMC_Dataset $))$; // DS indicates the Dataset

RFP $\leftarrow$ rf.merge $([$ rows, cols].isNull ()$)$;

rf.columns.metrics(Veriify_Columns);

rf.weight_score $>=0 \& \&$ rf.weight_score $\leq 1 \rightarrow$ Lbl='Low'; // Lbl indicates Label

rf.weight_score $>=2 \& \&$ rf.weight_score $\leq 5 \rightarrow$ Lbl='Med'; // Med indicates Medium Level

rf.weight score $>=6 \& \&$ rf.weight ${ }^{\text {score } \leq 10} \rightarrow$ Lbl='High';

5. Specify the number of dataset rows need to visualize to the users for verification.

6. Check if any null values present into the given dataset.

\section{Pseudocode:}

rf.head_display(100);

rf.is_null().if_any();

7. Perform reverse_mapping with all data extracted from the dataset.

8. Return the reverse_mapping results to the next process of training and modeling.

Pseudocode:

Rev_Map $\leftarrow$ reverse_mapping(rf.columns $(5))$;

return Rev_Map;

\section{B. Data Modeling and Prediction}

The data modeling and prediction logics depends on the data training and classification principles, in which the dataset pre-processing stage is completed, the next level of manipulation is called data training and model creation. For this the processed data is accumulated from the pre-processing procedure and raise a loop from 
initial data fond in the array to the last value found into the dataset. These kind of processing epochs are measured with respect to the proposed approach INNM mean ratio as well as the modeling procedures are created based on the training values. The created model is cross-validated with certain random rainfall records acquired from the trained dataset and the user can customize the cross-validation samples according to their testing needs. The model is cross-checked with the samples randomly acquired from the dataset and the resulting accuracy is emulated based on the samples provided for verification of the model. Once these model creation and cross-validation processes are done, the next level is the dynamic testing process, in which the live records can be input to the created model to test the respective values based on the INNM procedures to attain the high accurate results in outcome as well as the resulting section clearly proves this process in an efficient manner. The following algorithm, Algorithm-2 demonstrates the complete process flow of the data model creation and testing stages in detail with proper Pseudocode.

\section{Algorithm-2: Model Creation and Testing}

Input: Pre-processed data from Algorithm-1

Output: Trained Model, Prediction and Accuracy

1. Create a multi-dimensional array for data model processing.

2. Identify the pre-processed stage number of rows and columns and create an array index accordingly.

3. Raise a For loop to process total number of extracted pre-processed data, from 0 to n-1

4. Model Created with training data samples.

\section{Pseudocode:}

from(sk_learn.model.selection).import train.split;

$\mathrm{X} \leftarrow$ Rainfall_Data[drop('Lbl', axis=2)]; // axis indicates $(\mathrm{r}, \mathrm{c})$

$\mathrm{Y} \leftarrow$ Rainfall_Data $(\mathrm{X})$;

Res_Model $\leftarrow$ array_bound(list(X[Y]));

5. Create a new array variable to receive the resulting model rainfall data.

6. Input the dynamic rainfall data sample for testing the model with current value levels.

7. Initiate the created model to test the dynamic input of rainfall details with proper metrics.

8 . Return the prediction value as a result with proper accuracy ratio.

\section{Pseudocode:}

test_data $\leftarrow$ ['2/5/2021', 'Erode', 13.256, 17.256, 11.25]; $\quad$ Accuracy (\%)

pred $\leftarrow$ model_predict(test_data);

String Model_Accuracy(1);

Model_Accuracy $(0) \leftarrow$ pred;

Model_Accuracy $(1) \leftarrow$ Acc $($ pred $) / 100$;

return Model_Accuracy $(0,1)$;

\section{RESUltS AND DiscuSSIONS}

In this paper, a new hybrid machine learning principle is introduced called Intense Neural Network Mining (INNM), in which it provides a high factor resulting ratio with respect to Chennai-RMC weather dataset. The proposed approach is implemented by using one of the powerful machine learning strategic tool called Python Open Source Jupyter Notebook. The proposed approach of INNM produces the output range of $96.5 \%$ and the error rate coverage is so least at the range of $0.04 \%$. The following figure, Fig-2 illustrates the impact of proposed integration algorithm, in which the logic of Rapid Miner concept is included in order to avoid the data error values and provides the exact and actual data for processing as well as classification. Rainfall (mm) $\mathrm{g}$ provides highest accuracy range in results based on different rainfall ranges and the foll _ _ _ _ 2 shows the rainfall prediction accuracy range with and without pre-processing logic. The values clearly propagates the logic of pre-processing and its importance, in which the data prediction accuracy ranges are deviated from $2 \%$ to $4 \%$ of accuracy ranges as well as the form factor ratio is also decreased while manipulate the dataset without pre-processing procedure. This clearly demonstrates the importance of mining principle adaptation over the proposed approach as well as the figure ratio in y-axis (accuracy) indicates the level of prediction accuracy reduces with respect to rainfall increment while process the data without pre-processing, simultaneously check the dataset manipulation with pre-processing, the range improved while the rainfall level increases. 


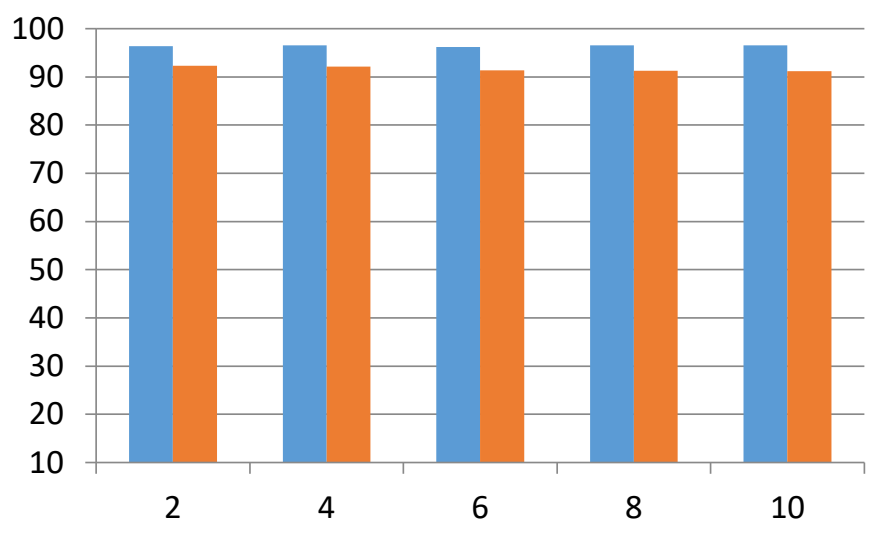

Fig.2 Accuracy Level Estimations with respect to Mining Principles

The following figure, Fig-3 illustrates the graphical representation of the proposed approach INNM accuracy levels over rainfall data acquisition range versus the relevant bit error ratio. This figure shows the prediction accuracy levels of the different ranges of data collection with respect to error ratio, in which the $\mathrm{x}$-axis shows the rainfall record acquiring ratio in bits and the respective bit error ratio is portrayed in the y-axis. For better understanding of the bit error rate, the prediction accuracy range is illustrated from the levels between 0 and 1 .

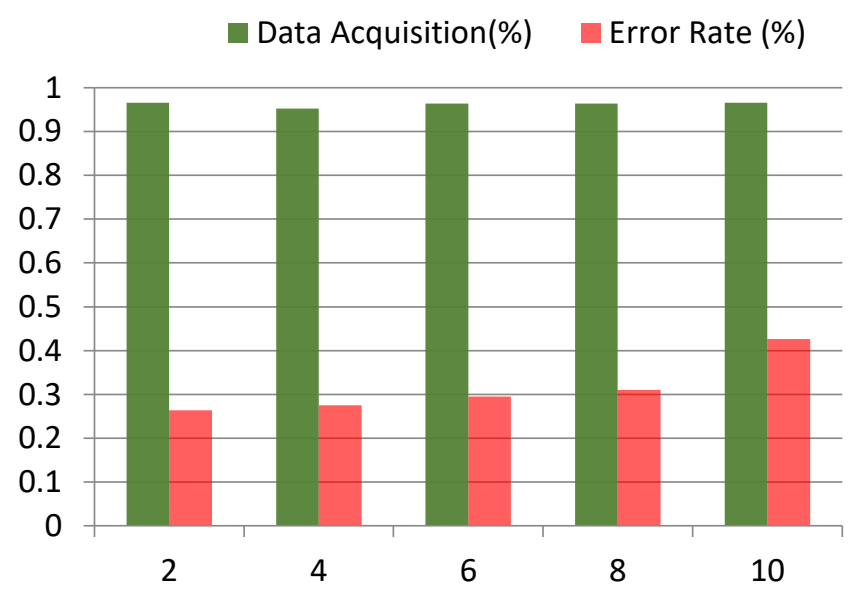

Rainfall (mm)

Fig.3 Rainfall Prediction Accuracy vs. Error Rate

\section{CONCLUSION AND Future SCOPE}

This paper concentrates more on rainfall predictions to preserve the water source as well as save many people from the disasters with respect to new machine learning approach. In this paper, a novel hybrid machine learning strategy is introduced called Intense Neural Network Mining (INNM), in which it integrates two efficient algorithms to manipulate the rainfall data and provides a highest range of accuracy in results. The two associated algorithms are Back Propagation Neural Network (BPNN) and Rapid Miner (RM) logic. These two algorithms are properly associated with the proposed machine learning approach to improves the accuracy level around $96.5 \%$ with least error ratio of $0.04 \%$ and the resulting section figures, Fig-1 and Fig-2 portrays that in a clear manner. In this paper, the dataset provided by Chennai-Regional Meteorological Centre (C-RMC) is utilized to cross-validate the proposed model and the resulting accuracy is attained accordingly. For all the proposed approach of INNM is good enough to predict the rainfall conditions effectively and proves the resulting performance through its accuracy levels. 
This proposed approach of Intense Neural Network Mining is further enhanced by means of adding some deep learning procedures in association with classification logic such as AdaBoost Classifier to improve the proposed approach accuracy levels from $96.5 \%$ to more. This proposed approach INNM clearly describes regarding the successful rainfall data prediction with good accuracy level but the appliance of this deep learning principles improves the data processing level in rich manner with proper time estimations.

\section{REFERENCES}

Yuelong Zhu, Jun Feng, Le Yan, Tao Guo and Xiaodong Li, "Flood Prediction Using Rainfall-Flow Pattern in Data-Sparse Watersheds", IEEE, Volume: 8, DOI: 10.1109/ACCESS.2020.2971264, 2020.

Zening Wu, Yihong Zhou and Huiliang Wang, "Real-Time Prediction of the Water Accumulation Process of Urban Stormy Accumulation Points Based on Deep Learning", IEEE, Volume: 8, DOI: 10.1109/ACCESS.2020.3017277, 2020.

Chaw Chaw Khaing and Thin Lai Lai Thein, "Prediction of Rainfall Based on Deep Learning and Internet of Things to Prevent Landslide", IEEE 9th Global Conference on Consumer Electronics (GCCE), 2020.

Xiaobo Zhang, Sachi Nandan Mohanty, et al., "Annual and Non-Monsoon Rainfall Prediction Modelling Using SVR-MLP: An Empirical Study From Odisha", IEEE, Volume: 8, DOI: 10.1109/ACCESS.2020.2972435, 2020.

Mohd Imran Khan and Rajib Maity, "Hybrid Deep Learning Approach for Multi-Step-Ahead Daily Rainfall Prediction Using GCM Simulations", IEEE, Volume: 8, DOI: 10.1109/ACCESS.2020.2980977, 2020.

Guohui Li, Wanni Chang and Hong Yang, "A Novel Combined Prediction Model for Monthly Mean Precipitation With Error Correction Strategy", IEEE, Volume: 8, DOI: 10.1109/ACCESS.2020.3013354, 2020.

Anik Naha Biswas, Yee Hui Lee and Shilpa Manandhar, "Analysis of GPS Gradient Parameters for Rainfall Prediction", IEEE USNC-CNC-URSI North American Radio Science Meeting,

DOI: 10.23919/USNC/URSI49741.2020.9321610, 2020.

Kumar Vijay Mishra and Bhavani Shankar M. R., "Deep Rainrate Estimation from Highly Attenuated Downlink Signals of Ground-Based Communications Satellite Terminals", IEEE International Conference on Acoustics, Speech and Signal Processing, DOI: 10.1109/ICASSP40776.2020.9054729, 2020.

Eric Samikwa, Thiemo Voigt and Joakim Eriksson, "Flood Prediction Using IoT and Artificial Neural Networks with Edge Computing", IEEE International Conferences on iThings and GreenCom and CPSCom and SmartData and Cybermatics, DOI: 10.1109/iThings-GreenCom-CPSCom-SmartDataCybermatics50389.2020.00053, 2020.

Anjali Samad, Bhagyanidhi, Vaibhav Gautam, Piyush Jain, Sangeeta and Kanishka Sarkar, "An Approach for Rainfall Prediction Using Long Short Term Memory Neural Network", IEEE-ICCCA, 2020.

http://www.imdchennai.gov.in/dailyweekly.pdf 